\title{
Test System Impact on System Availability
}

\author{
Pau, L. F.
}

Published in:

I E E E Transactions on Aerospace and Electronic Systems

Link to article, DOI:

10.1109/TAES.1987.310856

Publication date:

1987

\section{Document Version}

Publisher's PDF, also known as Version of record

Link back to DTU Orbit

\section{Citation (APA):}

Pau, L. F. (1987). Test System Impact on System Availability. I E E E Transactions on Aerospace and Electronic Systems, AES-23(5), 625-633. https://doi.org/10.1109/TAES.1987.310856

\section{General rights}

Copyright and moral rights for the publications made accessible in the public portal are retained by the authors and/or other copyright owners and it is a condition of accessing publications that users recognise and abide by the legal requirements associated with these rights.

- Users may download and print one copy of any publication from the public portal for the purpose of private study or research.

- You may not further distribute the material or use it for any profit-making activity or commercial gain

- You may freely distribute the URL identifying the publication in the public portal

If you believe that this document breaches copyright please contact us providing details, and we will remove access to the work immediately and investigate your claim. 


\section{INTRODUCTION}

\section{Test System Impact on System Availability}

L.F. PAU, Senior Member, IEEE

Technical University of Denmark

The specifications are presented for an imperfect automatic test system (ATS) (test frequency distribution, reliability, false alarm rate, nondetection rate) in order to account for the availability, readiness, mean time between unscheduled repairs (MTBUR), reliability, and maintenance of the system subject to monitoring and test. A time-dependent Markov model is presented, and applied in three cases, with examples of numerical results provided for preventive maintenance decisions, design of an automatic test system, buffer testing in computers, and data communications.

Manuscript received August 1, 1984.

Author's address: Technical University of Denmark, Bldg. 348/EMI, DK 2800, Lyngby, Denmark.

0018-9251/87/0900-0625\$1.00 ㄷ 1987 IEEE
Much of the current debate around self-testing amounts to design system architectures (both in hardware and software) such that the unit under test (UUT) can be tested or monitored at selected points in time, by a monitoring and test system (ATS) subject to the same causes of hazards. If the ATS continuously monitors the UUT, the joint system (ATS + UUT) will have a reliability less than the smallest of the ATS and UUT reliabilities. If the ATS never controls the UUT, the failures of the latter may go unnoticed. However, the UUT failures may be mistaken for ATS failures, especially if the ATS reliability is not maximum. For cost reasons and others, it may not be justifiable to design the ATS with the highest possible reliability.

Many real life examples, especially from avionics, communications, and electronics, teach us that self-testing requires a well-balanced tradeoff between UUT reliability, ATS reliability, and the test strategy.

In terms of a sensitivity analysis, it is now especially dangerous to disregard the ATS reliability, when evaluating the system availability. The issues addressed are all related to the effects of an imperfect test system on the availability of the system under test, with its associated on-line monitoring and test system (ATS). The testing of the system/UUT is assumed to be a random checkout policy with a known probability distribution. The main imperfections accounted for are: 1) UUT reliability, 2) test system reliability, 3) false alarm rate of the test system, 4) nondetection rate of the test system, and 5) losses affecting the transmission of the alarms.

Moreover, independent of the technique used for failure diagnosis or detection, it is essential to investigate the effects of the maintenance and removal policies on this same system availability, in the presence of an imperfect monitoring and test system. Maintenance may in this context be restricted to repair, or to simple error correction, and the duration is characterized by a known probability distribution. The four classes of maintenance actions considered here are 1) on-site (or fast) repair after a failure has been detected by the test system, 2) off-site (or slow) repair after a failure has been detected by the test system, 3) withdrawal of UUT from service because of replacement, and 4) pulling down UUT for possible test after removal.

Finally, after unscheduled maintenance, repair, or error correction, which are here assumed to be without defects, the systems may be stored on-site or off-site. The same happens in case of withdrawal from service. The storage time distributions are supposed to be known. Preventive maintenance is carried out after a fixed duration (or number of cycles) $T$, also called life length.

No single general model is known to take into account the imperfections of the test and monitoring system. Only inspection errors in quality control have been considered [10], although a number of works have been dealing with models of preventive maintenance and 
system reliability $[2,5,8,15]$, or failure detection and diagnosis $[13,14]$ and automatic test systems [9].

The model proposed here is of wide applicability in terms of on-line test and monitoring systems, with obviously different orders of magnitude for all parameters involved: avionics $[1,11]$, telecommunications, control systems, manufacturing lines, computer systems, and integrated circuits [16]. Should the restrictions used here to deal with the time-dependent Markov model of a single-unit repairable system not hold, or should the online monitoring and testing not be random, there is literature to cover such specific extensions.

\section{OPERATING AND MAINTENANCE PROCEDURES}

These procedures are schematized in Fig. 1. We define $r_{1}$ as the probability of detecting correctly the system failure with an operating ATS, $r_{2}$ as the probability of assigning a faulty UUT to off-site repair, rather than on-site repair, $r_{3}$ as the probability of putting back into operational storage (condition $\mathrm{E}_{7}$ ) a system which was just pulled-down incorrectly, rather than testing it (condition $\mathrm{E}_{1}$ ), and $r_{4}$ as the probability that the ATS identifies correctly the UUT as being operational.

\section{A. System Or Unit Under Test (UUT)}

The system is assumed to operate according to specifications until one of the following circumstances happens:
1) The system undergoes a test or check-out (without removal), which is performed with an automatic test and monitoring system (ATS). During this test the UUT does not operate according to specifications and the test sequences are randomly distributed over time with a rate $\mu_{01}(t)$.

2) The system is improperly pulled down for accessability considerations due to other equipments; it is not left in pulled-down position (no holding time), and either tested by the ATS or assigned to on-site storage in a random way.

3) The system is withdrawn from service because of replacement, or of aging, and goes to off-site storage without test.

Finally, the UUT is assumed to operate correctly again after the end of the test sequence, assuming that no failure has been detected.

\section{B. Test And Monitoring System (ATS)}

1) The test and monitoring system tests the UUT, while it operates, at the rate $\mu_{01}(t)$, and tests also the UUT when it has been improperly pulled down.

2) If no failure is detected by the ATS, either after checkout or removal, the system is put back into operational status.

3) The test and monitoring unit will deliver a negative output, or alarm, in either of the following 2 cases:

a) The ATS itself has failed and the ATS has the reliability function $G(t)$ (See [20]).

b) The ATS has not failed, but either the UUT is

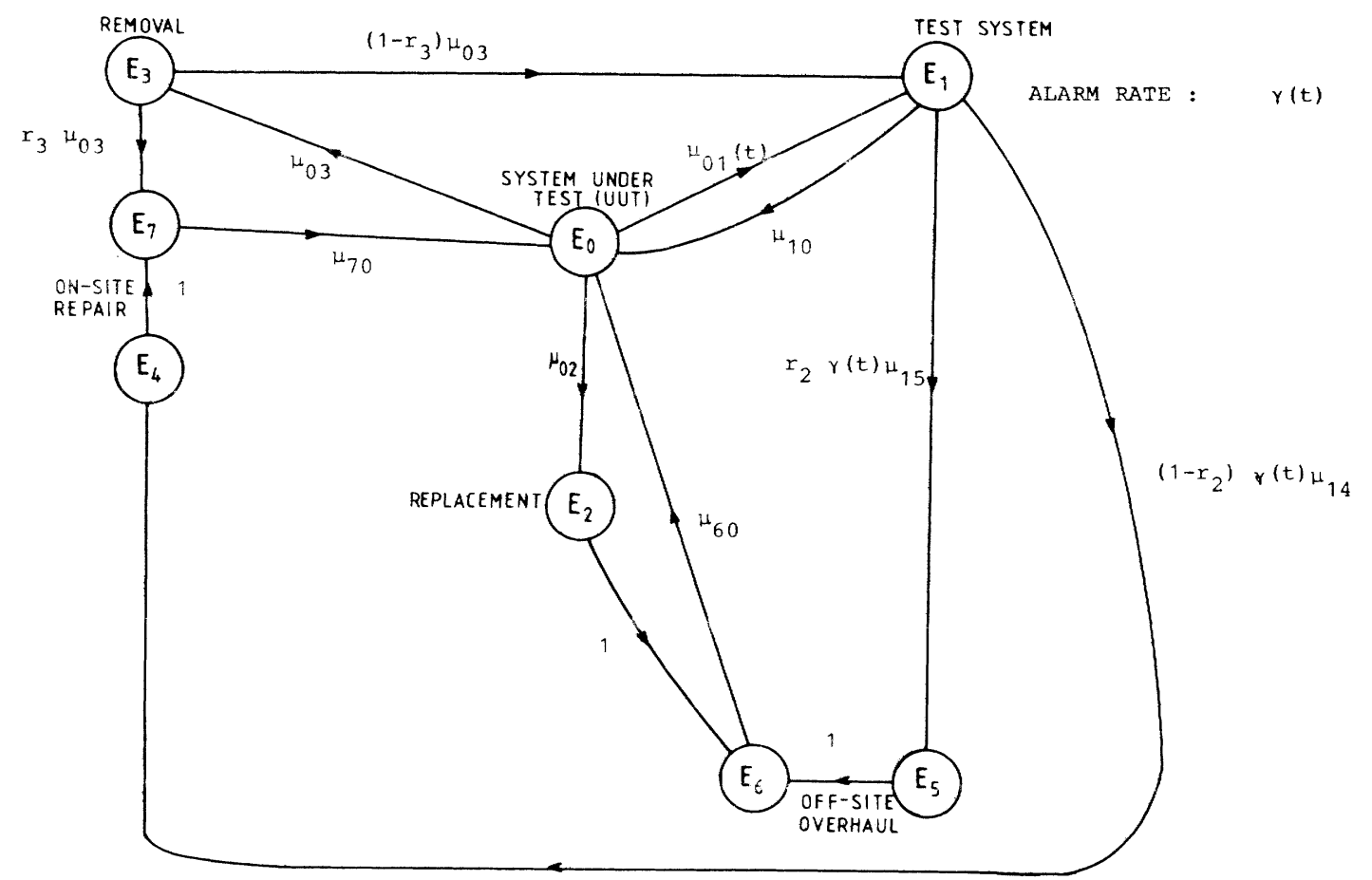

Fig. 1. Markov state diagram for UUT and its test system, with corresponding maintenance actions: $\gamma(t)=1-G(t)+G(t)\left(1-r_{4}\right) F(t)+$ $G(t) r_{1}(1-F(t))$, and $\mu_{10}(t)=1-\gamma(t)\left[\left(1-r_{2}\right) \mu_{14}+r_{2} \mu_{15}\right]$. 
operating correctly, and the operating ATS has detected a failure (false alarm), or the UUT has a failure, and the ATS has detected it, although there is a nondetection probability; the UUT has the reliability function $F(t)$.

The two cases in 3)b) above are illustrated by the decision table for the ATS detection system, which relates the detected UUT condition with its true condition, in which (see Fig. 2)

$1-r_{1} \triangleq$ nondetection probability

$1-r_{4} \triangleq$ false alarm rate.

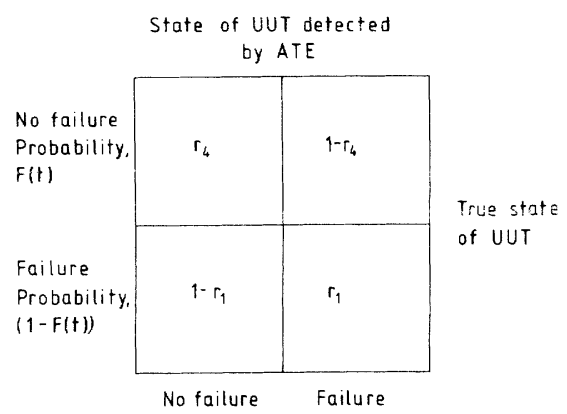

Fig. 2. Decision table for ATS detection problem for UUT.

Nondetection rate is $1-r_{1}$; false alarm rate is $1-r_{4}$; UUT reliability is $F(t)$.

\section{Maintenance And Repair}

If the ATS has delivered an alarm, the UUT will undergo either on-site (or fast) repair, or off-site (or slow) repair. After repair, the UUTs are stored in one of two possible inventories before being returned into service. No account is made of storage or maintenance induced failures.

\section{Remarks}

Please note that in the above procedure, and in the model of Section III, all operating and maintenance procedures are assumed to have no memory, which is often the case for electronic systems.

Also, we assume that the repair times are exponential, the inter-test times are Markovian, and all event types are mutually independent.

Should some specific applications not match these restrictive assumptions, a number of generalizations to more complex reliability problems can be found in the literature $[17,18,19]$.

\section{MODEL}

To describe the process outlined in Section II, we propose a continuous time Markov model, with timedependent coefficients and transition rates (see $[4,6]$ ).

State Probabilities (see Fig. 1): Let $p_{i}(t)$ be the probability for the system (ATS and UUT) to be in one of the following eight states:

$\mathrm{E}_{0}$ : System (UUT) in operational status; a failure is the condition $\overline{\mathrm{E}}_{0}$.

$\mathrm{E}_{1}$ : System under test without removal (UUT failed or not).

$E_{2}$ : System withdrawn from service because of replacement; goes at once to $\mathrm{E}_{6}$.

$E_{3}$ : System improperly pulled-down, with possible test after removal, or on-site storage.

$\mathrm{E}_{4}$ : On-site (fast) repair after negative test outcome.

$\mathrm{E}_{5}$ : Off-site (slow) overhaul after negative test outcome.

$E_{6}$ : Off-site storage of UUT in operational status (identical to $\mathrm{E}_{2}$ ).

$E_{7}$ : On-site storage of UUT in operational status.

Please note that the states $\mathrm{E}_{2}$ and $\mathrm{E}_{6}$ are identical computationally, though not when specifying the maintenance procedures.

Transition rates: We define the following transition rates, where all limits are for $\Delta t \rightarrow 0_{+}$:

Reliability

$\lambda_{U}(t)=\lim \operatorname{Pr}\left(\mathrm{E}_{0} \rightarrow \overline{\mathrm{E}}_{0}\right.$ during $\left(t, t+\Delta t\left(\mid \mathrm{E}_{0}\right.\right.$ at $\left.t\right) /$ $\Delta t$ represents the time-dependent failure rate of the UUT corresponding to $F(t)$.

$\lambda_{T}(t)=\lim$ Probability of an ATS failure during the interval $(t, t+\Delta t$, given it was operating at time $t) / \Delta t$ ).

Testing

$\mu_{01}(t)=\lim \operatorname{Pr}\left(\mathrm{E}_{0} \rightarrow \mathrm{E}_{1}\right.$ during $\left(t, t+\Delta t\left(\mid \mathrm{E}_{0}\right.\right.$ at $\left.t\right) /$ $\Delta t$ represents the rate at which the UUT is being connected to the ATS without pulldown.

$\mu_{10}(t)=\lim \operatorname{Pr}\left(\mathrm{E}_{1} \rightarrow \mathrm{E}_{0}\right.$ during $\left(t, t+\Delta t\left(\mid \mathrm{E}_{1}\right.\right.$ at $\left.t\right) /$ $\Delta t$ represents the rate at which the UUT is being put back into service in case of positive test outcome (no failure) (see Section IIB 3).

$\mu_{02}(t)=\lim \operatorname{Pr}\left(\mathrm{E}_{0} \rightarrow \mathrm{E}_{2}\right.$ during $\left(t, t+\Delta t\left(\mid \mathrm{E}_{0}\right.\right.$ at $\left.t\right) /$ $\Delta t$ represents the rate at which an operating UUT is being removed from service for replacement, or because of aging, or because of preventive maintenance at time $T ; \mu_{02}$ is time dependent because of aging. $\mu_{03}=\lim \operatorname{Pr}\left(\mathrm{E}_{0} \rightarrow \mathrm{E}_{3}\right.$ during $\left(t, t+\Delta t\left(\mid \mathrm{E}_{0}\right.\right.$ at $\left.t\right) /$ $\Delta t$ represents the rate at which an operational system is being improperly pulled down.

Maintenance

$\mu_{14}=\lim \operatorname{Pr}\left(\mathrm{E}_{1} \rightarrow \mathrm{E}_{4}\right.$ during $\left(t, t+\Delta t\left(\mid \mathrm{E}_{1}\right.\right.$ at $\left.t\right) /$ $\Delta t$ represents the rate at which a system under test, eligible for maintenance due to the outcome of the test, can be repaired onsite (fast repair).

$\mu_{15}=\lim \operatorname{Pr}\left(\mathrm{E}_{1} \rightarrow \mathrm{E}_{5}\right.$ during $\left(t, t+\Delta t\left(\mid \mathrm{E}_{1}\right.\right.$ at $\left.t\right) /$ $\Delta t$ represents the rate at which a system under test, eligible for maintenance due to 
the outcome of the test, can be repaired offsite (slow repair).

$\mu_{26}=1$ expresses that all UUT removed for replacement go to off-site storage, as $E_{2}$ and $\mathrm{E}_{6}$ are identical states computationally.

$\mu_{60}=\lim \operatorname{Pr}\left(\mathrm{E}_{6} \rightarrow \mathrm{E}_{0}\right.$ during $\left(t, t+\Delta t\left(\mid \mathrm{E}_{6}\right.\right.$ at $\left.t\right) /$ $\Delta t$ represents the rate at which an operational system stored off-site is being put back into service.

$\mu_{70} \cdot \Delta t=\lim \operatorname{Pr}\left(\mathrm{E}_{7} \rightarrow \mathrm{E}_{0}\right.$ during $\left(t, t+\Delta t\left(\mid \mathrm{E}_{7}\right.\right.$ at $\left.t\right) /$

$\Delta t$ represents the rate at which an operational system stored on-site is being put back into service.

\section{A. Exogeneous Probabilities}

Due to reliability considerations, or in connection with the maintenance organization, the following probabilities are defined:

$F(t)=\exp \left(-\int_{0}^{t} \lambda_{U}(x) d x\right):$ UUT reliability

$G(t)=\exp \left(\int_{0}^{t} \lambda_{T}(x) d x\right):$ ATS reliability.

One can then compute the probability $\gamma(t)$ for the test and monitoring unit to deliver an alarm, based on the outcomes listed in Section IIB:

$\gamma(t)=\operatorname{Pr}($ ATS output $=$ alarm at time $t)$

$\gamma(t)=1-G(t)+G(t)\left(1-r_{4}\right) F(t)$

$$
+G(t) r_{1}(1-F(t)) \text {. }
$$

Also, because the transition from $E_{1}$ back to operational status $E_{0}$ can only happen if the output of the test and monitoring system is positive (no alarm), the actual transition rate $\mu_{10}(t)$ is

$\mu_{10}(t)=1-\gamma(t)\left[\left(1-r_{2}\right) \mu_{14}+r_{2} \mu_{15}\right]$

where the second factor is the combined transition rate from $E_{1}$ to $E_{4}$ or $E_{5}$.

\section{B. State Evolutions}

The evolutions of the state probabilities $p_{i}(t)$ are governed by the equations (see Fig. 1):

$$
\begin{aligned}
\frac{d p_{0}}{d t}= & -\left(\mu_{01}(t)+\mu_{02}(t)+\mu_{03}\right) p_{0}(t) \\
& +\mu_{10}(t) p_{1}(t)+\mu_{60} p_{6}(t)+\mu_{70} p_{7}(t) \\
\frac{d p_{1}}{d t}= & -\left[\mu_{10}(t)+\left(1-r_{2}\right) \gamma(t) \mu_{14}\right. \\
& \left.+r_{2} \gamma(t) \mu_{15}\right] p_{1}(t) \\
& +\mu_{01}(t) p_{0}(t)+\left(1-r_{3}\right) \mu_{03} p_{3}(t) \\
\frac{d p_{1}}{d t}= & -p_{1}(t)+\mu_{01}(t) p_{6}(t) \\
& +\left(1-r_{3}\right) \mu_{03} p_{3}(t)
\end{aligned}
$$

\section{Initial Conditions}

Assuming both the UUT and ATS to start an operational life at $t=0$, and the states $\mathrm{E}_{1}-\mathrm{E}_{7}$ to be empty:

$p_{0}(0)=1 ; \quad p_{i}(0)=0, \quad i=1, \ldots, 7$.

\section{Ergodicity}

The above process is ergodic if and only if there exists one irreducible closed subset of positive persistent states, and if all of these states are aperiodic. It can be observed in practice that neither this criteria, nor the following are fulfilled in all cases:

$\lim _{t \rightarrow+\infty} \frac{d p_{i}}{d t}=0, \quad i=0, \ldots, 7$, independently of $p_{i}(0)$.

This lack of strong global ergodicity is essentially due to the imperfections of the test equipment, and to $\mu_{02} \neq 0$.

Ergodicity, as well as singularity conditions, are of secondary importance here, because we are only interested in finite horizon numerical solutions; this finite horizon is equal to the preventive maintenance period $T$.

One may easily compute, if needed, the ergodic coefficient of the stochastic process, and follow its time variations, and especially its convergence towards zero, in case of weak ergodicity ([6]).

\section{E. Mean Time Between Unscheduled Repairs}

Because of the preventive maintenance actions at time $T$, all unscheduled repairs and the role played by the test equipment, it is interesting to compare the mean time between failures (MTBF) and the mean time between unscheduled repairs (MTBUR) of the system under test:

$$
\begin{aligned}
\operatorname{MTBF}(T) & =\int_{0}^{T} F(t) d t \\
& =\int_{0}^{T}\left(\exp \left(-\int_{0}^{t} \lambda_{U}(x) d x\right) d t\right. \\
\operatorname{MTBUR}(T) & =\int_{0}^{T} p_{0}(t) d t
\end{aligned}
$$


where $p_{0}(t)$ is the solution of the stochastic process III B. The quantity $\operatorname{MTBF}(T)$ for a Weibull reliability process is studied in [12].

\section{APPLICATION A. PREVENTIVE MAINTENANCE DECISIONS}

\section{A. Criteria Applying To Preventive Maintenance}

One of the following maintenance decision rules can be considered [5]:

1) Overhaul or replace the system if it is found to be in a state of deterioration exceeding a control limit.

2) Minimize the cost per service time unit, when the cost of replacement or overhaul and the costs of being in the various degraded states are taken into account (including inspection costs).

3) Maximize the time between replacements or overhauls subject to the assumption that the reliability does not exceed some upper limit.

4) Minimize the life cycle costs per item, including procurement, testing, maintenance, inspection, and operations.

\section{B. Cost Minimization Of The Decision Between Unscheduled On-Site Repair And Off-Site Overhaul}

Assume that the test equipment activates an alarm at time $t, 0 \leq t \leq T$; the decision is between

$\mathrm{E}_{4}$ : on-site repair at cost $a$,

$\mathrm{E}_{5}$ : off-site overhaul at cost $A, A \geq a$.

The point is that, if preventive maintenance (i.e., overhaul) is mandatory at time $t=T$, one may want to avoid having many repairs when an early overhaul would extend the subsequent planned operational life of the system [3]. Repair actions alone will not extend this planned operational life of the system. In case of on-site repair, this incremental cost $A$ should be spread over MTBUR $(t+T)$ because of the new extended authorized residual life $T$ and the termination at time $t$ of the former one. The maintenance strategy proposed here, as related to the operations described in Section II, is to select between $\mathrm{E}_{4}$ or $\mathrm{E}_{5}$ the decision which minimizes the incremental maintenance costs per unit of authorized residual life:

$\mathrm{E}_{4}$ : on-site repair at time $t$ if alarm at $t$ and

$$
\frac{a}{\operatorname{MTBUR}(T)} \leq \frac{A}{\operatorname{MTBUR}(t+T)}
$$

$\mathrm{E}_{5}$ : off-site overhaul at time $t$, if alarm at $t$ and

$$
\frac{a}{\operatorname{MTBUR}(T)}>\frac{A}{\operatorname{MTBUR}(t+T)} .
$$

The probability $r_{2}$ becomes the ratio of the probability of the decision $E_{5}$, to the probability of the decision $E_{4}$, as reflected by the values of $p_{5}(t)$ and $p_{4}(t)$. Numerical solutions may be obtained by forward integration, for specified values of $\mu_{01}(t), \mu_{02}(t), F(t)$, and $G(t)$.

\section{Examples:}

1) $\mu_{01}(t)$, test rate, is specified equal to zero over subintervals of $[0, T]$, corresponding to the unavailability of an on-site ATS.

2) $\mu_{02}(t)$, removal rate, is specified equal to zero over subintervals of $[0, T]$, for example during the contractual life of the equipment.

3) $F(t)$ is a Weibull failure distribution, and $G(t)=$ 1 ; numerical results are obtained in [12].

\section{Partial Conclusion}

Case studies of this application A, show that the ATS performances have very significant impact on the performance criteria values of the decision reached according to the criteria of Sections IVA and B. One might even sometimes claim that preventive maintenance makes sense or not, only if the ATS meets some reliability goals, which make that preventive policy visible.

\section{APPLICATION B. SPECIFICATION AND DESIGN OF TEST SYSTEMS}

\section{A. Introduction}

The problem of specifying jointly the reliability $G(t)$, testing rate $\mu_{01}(t)$, false alarm rate $\left(1-r_{4}\right)$, and nondetection rate $\left(1-r_{1}\right)$ of a test and monitoring system is essential for the overall operational availability and readiness of many systems operating as described in Section I. Moreover, these imperfections have a crucial effect on the preventive maintenance policy.

A case example shows how the model of Section III may help in selecting the above parameters, and carry out a sensitivity analysis. The extreme values of the availability $p_{0}(t)$, as well as other probabilities can also be obtained, and another design criteria is the $\operatorname{MTBUR}(T)$.

It is essential to find out how much more reliable than the UUT the ATS must be, or if the ATS imperfections make preferable a random maintenance policy without any test system. Another concern is not to maximize too much the rate $\mu_{01}(t)$ at which the monitored system is being tested, because of side-effects related to UUT availability and also to ATS reliability and cost.

\section{B. Standard Case}

A case is selected, corresponding to rather low reliabilities and low performances of the ATS, to enhance the interaction aspects with the system under test: 


\begin{tabular}{|c|c|c|}
\hline Reliability & Test & \\
\hline$\lambda_{U}=0,05$ & $r_{1}=0,8$ & \\
\hline$\lambda_{T}=0,025$ & $r_{4}=0,8$ & \\
\hline$T=1$ & $\Delta t=0,01 \quad \mu_{01}=0,1$ & \\
\hline \multicolumn{3}{|l|}{ Maintenance } \\
\hline$r_{2}=0,6$ & $\mu_{14}=0,1$ & $\mu_{15}=0,03$ \\
\hline \multicolumn{3}{|l|}{$r_{3}=0,15$} \\
\hline$\mu_{02}=0,001$ & $\mu_{70}=0,1$ & $\mu_{60}=0,1$ \\
\hline$\mu_{03}=0,001$ & & \\
\hline
\end{tabular}

TABLE I

\begin{tabular}{ll}
\hline \multicolumn{1}{c}{$\begin{array}{c}\text { Numerical } \\
\text { Results Standard Case }\end{array}$} & \multicolumn{1}{c}{$\begin{array}{c}\text { Max } / \text { Min } \\
\text { Values }\end{array}$} \\
\hline Availability $p_{0}(t)$ & $1.0 / 0.593$ \\
Testing probability $p_{1}(t)$ & $0.1 / 0.0596$ \\
Alarm rate $\gamma(t)$ & $0.959 / 0.234$ \\
MTBF $(T)$ & 0.194 \\
MTBUR $(T)$ & 0.730 \\
$F(T)$ & 0.00674 \\
$G(T)$ & 0.0821 \\
On-site repair $p_{4}(t)$ & $0 . / 0.144$ \\
On-site storage $p_{7}(t)$ & $0 . / 0.0135$ \\
Off-site repair $p_{5}(t)$ & $0 . / 0.0649$ \\
Off-site storage $p_{6}(t)$ & $0 . / 0.0103$ \\
\hline
\end{tabular}

Whereas most state probabilities have monotonous evolutions ( $p_{0}$ decreasing; $p_{3}, p_{4}, p_{5}, p_{6}, p_{7}=$ increasing), it is extremely interesting to observe that the testing probability $p_{1}(t)$ peaks at an intermediate stage $(t=37)$, and remains around $p_{1}=0,06$ until $t=T$. The replacement probability peaks also, and remains level thereafter.

\section{Sensitivity Analysis}

This analysis is restricted here to single perturbations with respect to the standard case of Section V B, and the main results are displayed in Table II. Complete timedependent plots are not possible here for lack of space. For low ATS reliability, the testing probability $p_{1}(t)$ may have several oscillations. This also occurs if the testing rate $\mu_{01}(t)$ is high and close to 1 , where instabilities occur.

\section{Specification Of Recalibration Intervals}

The model of Section II is also appropriate if the test system is not resident close to the UUT, and represents a calibration system. The issue is here to optimize the rate $\mu_{01}(t)$ at which recalibration is carried out, with the alarms corresponding to measurements out of specifications, or to drift.

\section{E. Partial Conclusion}

It is necessary to carry out an exhaustive sensitivity analysis about the extreme values of the availability $p_{0}(t)$, versus the ATS specification parameters of Section V A,

TABLE II

Application B. Specification Of The Test System-Sensitivity Analysis

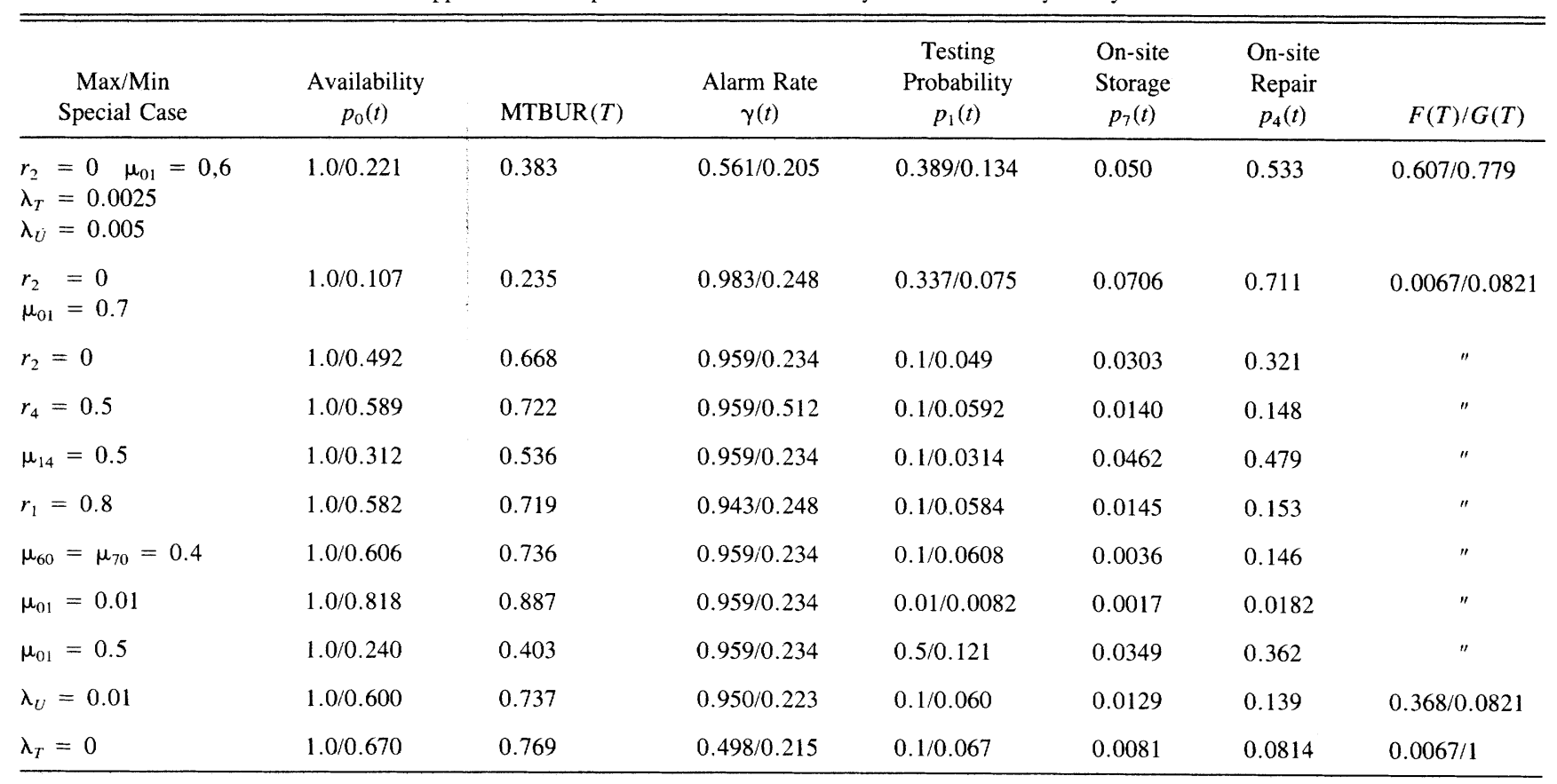


within the operational envelope of the UUT. These variations must be further reduced, with resulting narrowing down the specification parameter intervals, to let the test and alarm probabilities remain within bounds compatible with operational deployment policy.

\section{APPLICATION C. BUFFER TESTING IN COMPUTERS AND DATA COMMUNICATIONS}

\section{A. Introduction}

An interesting extension of the model in Section II, is where the condition $E_{0}$ is itself represented by a state diagram. This is the case when the UUT is a buffer of capacity $N$, subject to arrival and service processes as the ones encountered in computers and data communications. The test system then tests the packets arriving according to the arrival process, e.g., by an error detection code; error correction is either by a code (fast repair) or by requesting the retransmission (slow repair), and only correct or corrected packets enter the buffer.

\section{B. Buffer Model}

As a single approximation to the buffer space, we consider a single $M / M / 1-N$ queue with Poisson (1) arrival process, and independent identically distributed (IID) $(m)$ service process. If $q_{k}, k=0, \ldots, N$ is the total number of packets in the buffer number $k$, the steadystate distribution is

$q_{k}=\rho^{k}(1-\rho) /\left(1-\rho^{N+2}\right) \rho \triangleq 1 / m$.

The mean number of packets in the buffer is:

$\bar{N}=\sum_{k=0}^{N+1} k q_{k}$

and the mean waiting time is:

$W=\bar{T}-(1 / m)$

where $\bar{T}$ is the mean time spent in the buffer. The following formula are found in the steady-state case:

$$
\begin{aligned}
\bar{N}= & {\left[\rho-(N+2) \rho^{N+2}\right.} \\
& \left.+(N+1) \rho^{N+3}\right] /(1-\rho)\left(1-\rho^{N+2}\right) \\
\bar{T}= & (1 / m)+(\rho / m(1-\rho)) \\
& -(N+1) \rho^{N+1} /\left(m\left(1-\rho^{N+1}\right)\right) .
\end{aligned}
$$

The blocking probability, that is the probability that a packet arrives to a full buffer, after which it is lost, is

$q_{N+1}=\rho^{N+1}(1-\rho) /\left(1-\rho^{N+1}\right)$.

In the time-dependent case, the equations for the process are, for an arrival process Poisson $\left(L_{T}\right)$

$$
\frac{d q_{0}}{d t}=-L_{T} q_{0}+m q_{1}
$$

$$
\begin{aligned}
\frac{d q_{i}}{d t}= & L_{T}\left(q_{i-1}-q_{i}\right) \\
& +m\left(q_{i+1}-q_{i}\right), \quad i=1, \ldots, N-1
\end{aligned}
$$

$\frac{d q_{N}}{d t}=L_{T} q_{N-1}-m q_{N}$

with the initial condition $q_{0}(0)=1, q_{i}(0)=0$, $i=1, \ldots, N$.

\section{Failure Detection}

If the arriving packets are tested by the ATS prior to entering the buffer, the actual arrival process concerns only corrected or correct packets, with the actual arrival rate:

$L_{T}(t)=p_{0}(t)$

which depends on the states of the UUT and of the ATS. The measurement of $\bar{L}_{T}(t)$ is the subject of [7]. The original arrival process is Poisson $(L)$.

An alarm may now be initiated by the test system if there is a loss of information, that is, if some packets are lost. This supplements the other failure modes related to errors in the packets, or to the hardware of the UUT or ATS (see Section IIB3). As a consequence, the probability of alarm $\gamma(t)$ must incorporate the blocking probability for the $N$-buffer:

$\gamma_{N}(t)=\gamma(t)+q_{N+1}(t)$

Thus, the buffer condition will directly affect the test system depending on the traffic load, when $\gamma_{N}$ replaces $\gamma$ in the equations of Section III A.

\section{Numerical Example}

Because of numerical instability, the integration step for the joint system of differential systems of Sections IIIB and VIB must be reduced to $\Delta t / 10$.

\begin{tabular}{lcl}
\hline Reliability & \multicolumn{1}{c}{ Test } & Buffer \\
\hline$\lambda_{U}=0.001$ & $r_{1}=1-10^{-6}$ & $N=3$ \\
$\lambda_{T}=0.001$ & $r_{4}=1-10^{-8}$ & $L=0,2$ \\
$T=1 \quad \Delta t=0,01$ & $\mu_{01}=0,8$ & $m=0,2$
\end{tabular}

Maintenance

$$
\begin{array}{rlll}
r_{2} & =0 & & \\
r_{3} & =0 & \mu_{15}=0 & \mu_{14}=0,6 \\
\mu_{02}=0 & \mu_{70}=1 & \mu_{60}=0 \\
\mu_{03}=0 & &
\end{array}
$$


TABLE III

\begin{tabular}{ll}
\hline \multicolumn{1}{c}{ Numerical Results: Standard Case } & Min / Max Values \\
\hline Availability $p_{0}(t)$ & $0,112 / 1.0$ \\
MTBF $(T)$ & 0,304 \\
MTBUR $(T)$ & 0,875 \\
$F(T)$ & 0,905 \\
$G(T)$ & 0,905 \\
Alarm rate $\gamma_{N}(t)$ & $0,002 / 0,182$ \\
Testing probability $p_{1}(t)$ & $0.097 / 0,444$ \\
On-site storage $p_{7}(t)$ & $0 / 0,0078$ \\
On-site repair $p_{4}(t)$ & $0 / 0,783$ \\
Blocking probability $q_{N+1}(t)$ & $0 / 0,0217$ \\
Effective arrival rate $L_{T}(t)$ & $0,022 / 0,20$ \\
\hline
\end{tabular}

The numerical results (Table III) indicate rather high "maximum alarm rates" due to the values of $\lambda_{T}$ and $r_{1}$.

\section{E. Partial Conclusion}

The error-detection and error-correction schemes selected, with corresponding resulting alarm rates, must be adapted to buffer size and to the traffic arrival process.

\section{CONCLUSIONS}

This paper proposes a time-dependent Markov model describing jointly a system subject to random testing, and its associated test and monitoring system. The emphasis is on the imperfections of the test system, such as reliability, false alarm, and nondetection rate, and on their implications on the overall system availability, readiness and maintenance. It is shown through examples that the testing probability as well as the alarm rate, exhibit time-dependent oscillations, the extremes of which must be known. Tradeoffs must also be made between the system and test system reliabilities.

Application A deals with the application of the model to preventive maintenance decisions. Application B is a numerical analysis of the specification and design of a testing system. Application C covers buffer testing in computers and data communications. Here the operational state is itself subdivided, and the alarm rate is triggered also by the loss of information entering the buffer.

The main conclusions of this study are the following.

1) It is now possible to specify the ATS reliability in view of the UUT reliability and system availability, for given ATS failure detection performances.

2) It is necessary to take into account time-dependent evolutions of a number of test parameters in order to guarantee worst-case designs.

3) It is now possible to relate directly the test probability in self-testing systems to the overall system availability. 


\section{REFERENCES}

[1] Advisory Group for Aeronautical Research and Development (NATO) (AGARD) (1977)

Integrity in electronic flight control systems. AGARDograph, 224, Paris, 1977.

[2] Barlow, R., and Proschan, F. (1965) Mathematical Theory of Reliability. New York: Wiley, 1965, pp. 84-118.

[3] Chaudhuri, D., and Sahu, K.C. (1977) Preventive maintenance interval for optimal reliability of deteriorating systems. IEEE Transactions on Reliability, R-26, 5 (1977), 371-372.

[4] Dynkin, E.B., and Yushkevich, A.A. (1969)

Markov Processes: Theorems and Problems. New York: Plenum Press, 1969.

[5] Gertsbakh, I.B. (1977) Models of Preventive Maintenance. Amsterdam: North-Holland Publishing Co., 1977.

[6] Isaacson, D.L., and Madsen, R.W. (1976) Markov Chains: Theory and Applications. New York: Wiley, 1976.

[7] Iversen, V.B. (1976)

On the accuracy of measurements of time intervals and traffic intensities.

Presented at the 8th International Teletraffic Congress, Melbourne, Australia, 1976.

[8] Kaufman, A., Grouchko, D., and Cruon, R. (1975) Modèles Mathématiques Pour L'étude de la Fiabilité Des Systèmes.

Paris: Masson, 1975.

[9] Liguori F. (1975)

Automatic Test Equipment: Hardware, Software and Management.

New York: IEEE Press, 1974.

[10] Moskowitz, H., and Fink, R.K. (1977)

A bayesian algorithm incorporating inspection errors for quality control and auditing.

In M.F. Neuts (Ed.), Algorithm Methods in Probability, Studies in the Management Sciences, 7.

Amsterdam: North Holland Publishing Co., 1977.
[11] NASA (1975)

Systems reliability issues for future aircraft.

In Conference Proceedings 003, NASA, Aug. 18-20, 1975.

[12] Pau, L.F. (1970)

Révision générale et remise en état (Overhaul and repair). Technical Report, ENS Telécommunications, Paris, 1970.

[13] Pau, L.F. (1981)

Failure Diagnosis and Performance Monitoring.

New York: Marcel Dekker, 1981.

[14] Pau, L.F. (1982)

Failure diagnosis systems: An introduction.

Technical Report, IMSOR, Technical Univ., Denmark.

[15] Tsokos, C.P., and Shimi, I.N. (Ed.) (1977)

The Theory and Applications of Reliability With Emphasis On Bayesian and Nonparametric Methods.

New York: Academic Press, 1977.

[16] Murray, A.F., et al. (1983)

Self-testing in bit serial VLSI parts.

In Proceedings of the IEEE International Test Conference. New York: IEEE Press: 1983, p. 283.

[17] Karpinsky, J. (1986)

A multistate system under an inspection and repair policy. IEEE Transactions on Reliability, R-35, 1 (Apr. 1986), $76-78$

[18] Goel, Gupta, and Singh (1986)

Cost-benefit analysis of a 2 unit warm-standby system with inspection, repair and post repair.

IEEE Transactions on Reliability, R-35, 1 (Apr. 1986), $70-71$.

[19] Elsayedaly, N.A., and Aly, A.A. (1986)

Testability performance measurements of automatic diagnostic systems.

Technical Report, University of Oklahoma, Norman, Okla., 1986.

[20] Pliska, T.F., and Angus, J.E. (1979)

$\mathrm{BIT} /$ External test figures of merit and demonstration techniques.

Technical Report 79-309, Rome Air Development Center, Rome, N.Y., 1979.

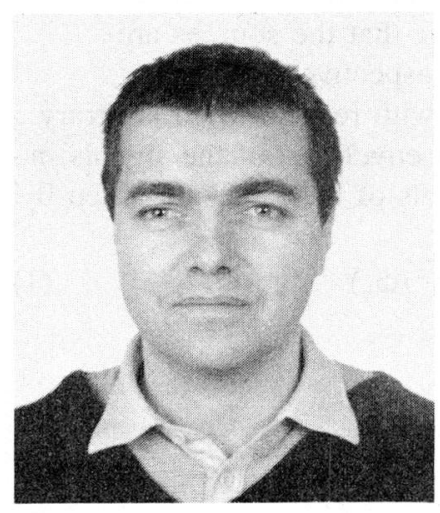

L.F. Pau (M'78, SM'78) was born in Copenhagen, Denmark. He holds an M.Sc. in aerospace electronics from E.N.S. Aéronautique et de l'Espace (Paris), as well as M.Sc., Ph.D., and D.Sc. from Paris University. He has been on the faculty of the E.N.S. Télécommunications (Paris), Massachusetts Institute of Technology (Cambridge, MA), University of Maryland. He was recently Senior scientist at the Battelle Memorial Institute. He is currently Research professor at the Technical University of Denmark. He has published 5 books and 100 reviewed papers in failure diagnosis, test systems, knowledge based systems, and signal classification. 